Relations industrielles

Industrial Relations

\title{
La contractualisation de la relation de travail, par Christian Bessy, Paris : LGDJ, 2007, 317 p., ISBN 978-2-275032-15-3.
}

\section{Urwana Coiquaud}

Volume 63, numéro 3, 2008

La relation d'emploi : état actuel de la question

The Employment Relationship: Current Research Avenues

La relación de empleo: estado actual de la cuestion

URI : https://id.erudit.org/iderudit/019106ar

DOI : https://doi.org/10.7202/019106ar

Aller au sommaire du numéro

Éditeur(s)

Département des relations industrielles de l'Université Laval

ISSN

0034-379X (imprimé)

1703-8138 (numérique)

Découvrir la revue

Citer ce compte rendu

Coiquaud, U. (2008). Compte rendu de [La contractualisation de la relation de travail, par Christian Bessy, Paris : LGDJ, 2007, 317 p., ISBN 978-2-275032-15-3.] Relations industrielles / Industrial Relations, 63(3), 576-578.

https://doi.org/10.7202/019106ar

Tous droits réservés @ C Département des relations industrielles de l'Université Laval, 2008
Ce document est protégé par la loi sur le droit d'auteur. L’utilisation des services d'Érudit (y compris la reproduction) est assujettie à sa politique d'utilisation que vous pouvez consulter en ligne.

https://apropos.erudit.org/fr/usagers/politique-dutilisation/ 
HRM with considerable gusto and research discipline. They have ordered the publication logically and utilized frequent illustrative techniques with models, tables and examples, all of which contribute to reader friendliness. There is less use of footnotes than in other texts, although academia might construe this in a critical way. In any objective summation, Strategy and Human Resource Management is considered to be a more than useful addition to what is a fairly crowded field of literary endeavour and is commended to students and interested practitioners alike.

Barry Plumb

Former Human Resource and Industrial Relations Executive The Gap, Australia

\section{La contractualisation de la relation de travail,} par Christian Bessy, Paris : LGDJ, 2007, 317 p., ISBN 978-2-275032-15-3.

La place du droit du travail dans l'économie capitaliste ainsi que l'encadrement des choix économiques par des dispositions de droit du travail ont souvent été questionnés tant chez les juristes que chez les économistes. Ce questionnement a pris tout son sens au début des années 1980 lorsque les bouleversements profonds de nos économies ont remis en cause les institutions en place. Certains économistes, critiques à l'égard de l'approche néo-classique, ont alors développé de nouveaux axes d'interprétation de ces phénomènes. C'est le cas de l'économie des conventions, dont est issu l'auteur de cet ouvrage, Christian Bessy, économiste du travail et spécialiste de l'analyse économique des institutions.

À partir de cette veine théorique exposée au chapitre 1, l'auteur examine les termes de la relation de travail d'aujourd'hui et cherche à expliquer le phénomène de contractualisation de cette relation. Il s'appuie dans cette démarche sur l'approche dite "interactive du droit », issue de la sociologie du droit, qui étudie l'activité sociale des acteurs orientée par les règles de droit. Ces règles, quels que soient leur source et leur caractère, seront mobilisées pour construire ou résoudre les situations sociales. Une telle approche conduit à observer l'utilisation routinière des outils juridiques, en l'occurrence les contrats de travail, faite par les acteurs. Parmi eux, certains jouent un rôle crucial, les « intermédiaires du droit », soit des professionnels du droit. Ces derniers ont la maîtrise des règles, des processus et de leur interprétation par les cours de justice. Ils peuvent donc guider la rédaction des contrats en anticipant les litiges sans que les parties à la relation de travail en comprennent toujours les tenants et les aboutissants. La prise en compte de cette activité des « intermédiaires du droit» conduit à relativiser en partie le rôle du droit traditionnel, celui issu de l'activité des pouvoirs publics et des tribunaux de juridiction supérieure, et à mettre en évidence une activité normative intermédiaire. L'auteur entend ainsi utiliser cette perspective pour mieux comprendre l'usage « instrumental », les « stratégies de contournement » du droit. Il pose à cet égard l'hypothèse d'un recul des relations plus coopératives et d'un affaiblissement des cadres collectifs comme facteur explicatif.

Christian Bessy entreprend donc les analyses qualitative et quantitative de plus de 400 contrats de travail, répartis sur plusieurs décennies (1970 à 2004), en provenance de plus de 300 entreprises issues de différents secteurs économiques. Il examine ainsi l'évolution des pratiques contractuelles 
et les transformations du droit du travail et de la gestion des ressources humaines, puis dégage de ce vaste horizon temporel les « standards rédactionnels » des contrats et leur évolution.

À partir de cette analyse l'auteur présente une typologie. Il se base sur quatre variables décrites aux chapitres $3,4,5,6$, soient les garanties en matière d'embauche, la subordination du salarié, la protection des actifs immatériels de l'entreprise et la responsabilisation du salarié, qu'il décline en différents indicateurs. Puis, il propose quatre catégories (dites « classes ») de contrats, classées selon le «degré croissant de complexité de leur structure contractuelle » (p. 180), degré qui permet d'indiquer la «densité de garantie contractuelle » recherchée par l'employeur.

Outre l'intérêt de démontrer la pluralité des relations de travail, cette typologie met en relief la diversité du rôle des parties à la relation. Dans le cadre de la première et seconde classe, le contrat est surtout utilisé comme un dispositif d'information et de clarification puisque la référence au statut collectif y est importante. Tandis que dans la classe 3 , qui se caractérise par une contractualisation «forcée » et une instrumentalisation du droit, il est surtout question d'asseoir dans le contrat l'autorité hiérarchique de l'employeur. Cette autorité, quoique présente dans la dernière catégorie de contrat (classe 4), l'est de façon plus atténuée car le salarié dispose d'une position plus avantageuse auprès de l'employeur, ce qui lui permet de négocier des contreparties (chapitre 7). Cette analyse est complétée par trois études de cas (chapitre 8) qui permettent à l'auteur de décrypter l'usage qui est fait du contrat au cours d'un litige. En effet, lors de la résolution des règlements des litiges, devant une juridiction de niveau inférieur, il n'est pas seulement fait usage de la normativité étatique (macro). D'autres sources de normativité, créées par la pratique, par un apprentissage collectif des acteurs, sont mobilisées (par exemple, le travail d'intermédiation des conseillers prud'homaux ou encore celui des syndicats). La reconnaissance de cette normativité devrait limiter l'usage stratégique du droit entre les mains des acteurs puisqu'elle n'est pas la seule mobilisée lors du processus de production des jugements. Dans les deux chapitres (9 et 10) qui suivent, l'auteur observe un double mouvement dans la transformation du contrat de travail : d'une part, les règles juridiques peuvent avoir des effets sur les pratiques contractuelles et, d'autre part, les mutations dans les modes de gestion peuvent faire évoluer les règles juridiques régissant le contrat de travail. Ce dernier est alors vu comme une institution qui se crée au carrefour de ces interactions complexes.

Il reste que dans ce contexte, la contractualisation des relations de travail conduit à un rapport de forces où l'employeur ressort le plus souvent gagnant. En l'absence de pouvoirs collectif et individuel de négociation, le salarié est soumis à une flexibilité de ses conditions de travail et à une responsabilisation accrue qui ne trouvent pas de justes contreparties dans la relation de travail; « un pur rapport de force en dehors de tout fondement légitime » (p. 298) dira l'auteur. Ce dernier voit dans cette évolution «le recul des supports collectifs de coopération dans l'entreprise et les difficultés d'émergence d'un nouveau modèle salarial» (p. 299). Ce «déficit institutionnel » creuse les inégalités et ouvre la voie à une redéfinition du rôle de l'État et des politiques publiques dans lequel la place du droit du travail est à redessiner et la protection sociale à assurer.

En conclusion, cet ouvrage sera utile à ceux et celles qui s'intéressent aux transformations contemporaines du travail et du droit du travail. Il offre un cadre conceptuel riche, complexe et original pour aborder le phénomène de 
la contractualisation de la relation de travail. En ce sens, il atteint les objectifs de la collection «droit et société » qui vise à « replacer le droit dans son tissu social ». Il reste que sa lecture peut s'avérer particulièrement exigeante en raison de l'examen pluridisciplinaire du phénomène (droit, économie et sociologie du droit) et de la diversité des méthodes employées pour cet examen. D'ailleurs, par souci de précaution, le lecteur devra prendre soin de replacer l'analyse livrée par l'auteur dans son contexte. Mais c'est là le tribut d'une recherche audacieuse, sur un phénomène complexe. Il reste que la lecture livrée par l'auteur de ce phénomène pave la voie à des études comparatives qui devraient s'avérer riches de sens et d'enseignements.

Urwana CoIQuaud HEC Montréal

\section{What Workers Say: Employee Voice in the Anglo-American Workplace,} edited by Richard B. Freeman, Peter Boxall and Peter Haynes, Ithaca: Cornell University Press, 2007, 244 pp., ISBN 978-0-8014-7281-7.

When this book arrived I eagerly read a few of the key chapters. Many of the contributors are colleagues and friends that I know and respect so I welcomed an opportunity to praise their work. But this book, while reporting useful survey results on workers attitudes and conditions with regard to voice at work, has some serious problems of conception and execution.

The inspiration for What Workers Say is the Workers Representation and Participation Survey (WRPS) originally carried out by Richard Freeman and Joel Rogers and reported in What Workers Want (1999, updated in 2006). That survey was remarkable for its finding that nearly all American workers wanted some form of collective representation in the establishment of their conditions of work. Some $32 \%$ of nonunion workers wanted a union. Most of the rest wanted "an organization with more limited independence from management." Only $7 \%$ wanted "no organization."

Unfortunately, in my opinion, What Workers Say does not effectively move forward the agenda of revealing the general failure of society to provide working people with collective representation of the sort that they want and to which international human rights standards say they are entitled. Instead, although there is some discussion of (so-called) "nonunion forms of representation," What Workers Say focuses primarily on traditional union density and the unfulfilled demand for traditional union representation. Alternative voice mechanisms are discussed to an extent but, for the most part, are conceived of as employerinstituted competition for independent unions rather than as alternatives that employees might choose instead of conventional adversarial unionism. There is no clarity of awareness of the important distinction between independent local unionism and employer-dominated employee representation schemes. Into the nonunion conceptual basket goes everything from management briefing schemes to joint consultative committees to whatever the researcher considers to be unconventional worker representation.

The contributors to What Workers Say collected similar (but not exactly the same) data from Anglo-American countries other than the United States. In the United Kingdom, Ireland, Australia, and New Zealand fresh surveys modeled generally on the Freeman and Rogers study were carried out. In Canada the researchers (Michele Campolieti, Rafael Gomez and Morley Gunderson) mined further the database assembled 